\title{
Thermodynamics and kinetic modeling of the homogeneous gas phase reactions of the oxidative coupling of methane
}

\section{Citation for published version (APA):}

Geerts, J. W. M. H., Chen, Q., Kasteren, van, J. M. N., \& Wiele, van der, K. (1990). Thermodynamics and kinetic modeling of the homogeneous gas phase reactions of the oxidative coupling of methane. Catalysis Today, 6(4), 519-526. https://doi.org/10.1016/0920-5861\%2890\%2985047-R

DOI:

10.1016/0920-5861\%2890\%2985047-R

Document status and date:

Published: 01/01/1990

\section{Document Version:}

Publisher's PDF, also known as Version of Record (includes final page, issue and volume numbers)

\section{Please check the document version of this publication:}

- A submitted manuscript is the version of the article upon submission and before peer-review. There can be important differences between the submitted version and the official published version of record. People interested in the research are advised to contact the author for the final version of the publication, or visit the $\mathrm{DOI}$ to the publisher's website.

- The final author version and the galley proof are versions of the publication after peer review.

- The final published version features the final layout of the paper including the volume, issue and page numbers.

Link to publication

\section{General rights}

Copyright and moral rights for the publications made accessible in the public portal are retained by the authors and/or other copyright owners and it is a condition of accessing publications that users recognise and abide by the legal requirements associated with these rights.

- Users may download and print one copy of any publication from the public portal for the purpose of private study or research.

- You may not further distribute the material or use it for any profit-making activity or commercial gain

- You may freely distribute the URL identifying the publication in the public portal.

If the publication is distributed under the terms of Article 25fa of the Dutch Copyright Act, indicated by the "Taverne" license above, please follow below link for the End User Agreement:

www.tue.nl/taverne

Take down policy

If you believe that this document breaches copyright please contact us at:

openaccess@tue.nl

providing details and we will investigate your claim. 
THERMODYNAMICS AND KINETIC MODELING OF THE HOMOGENEOUS GAS PHASE REACTIONS OF THE OXIDATIVE COUPLING OF METHANE.

J.W.M.H. Geerts, Q. Chen, J.M.N. van Kasteren, K. van der Wiele

Department of Chemical Technology, Eindhoven University of Technology, P.O. Box 513, $5600 \mathrm{MB}$ Eindhoven (The Netherlands)

\section{SUMMARY}

Thermodynamic calculations concerning methane activation have shown that in the equilibrium state hydrocarbons are only present in very small quantities. This is in contrast to experiments carried out under kinetically determined conditions, where considerable amounts of ethane and ethylene are formed via a radical mechanism. A computer model has been developed to simulate and analyze networks of those radical reactions operative in the oxidative coupling of methane.

\section{INTRODUCTION}

A new promising method for the production of ethylene is the partial oxidation of methane in the presence of a catalyst. To analyze the importance of radical reactions in this process, non-catalytic experiments were carried out in a micro flow reactor and simulated with a. computer model. The model covers 164 elementary reactions, each described by an Arrhenius-like type kinetic equation. These equations are combined with an one dimensional reactor model, assuming plug flow and isothermal conditions in first instance. Axial temperature profiles are a built-in option.

\section{RESULTS AND DISCUSSION}

Thermodynamic calculations

The equilibrium composition of pure methane pyrolysis and that of pyrolysis in combination with methane oxidation have been calculated. The formation of elementary carbon was excluded, for reasons of simplicity. Figure 1 shows the conversion and yields (= the product of conversion and selectivity) of methane pyrolysis. The selectivities and yields have been defined on carbon bases. It is very clear that ethylene is the main hydrocarbon product at high temperatures in the chemical equilibrium state. However, the methane conversion is limited. If methane pyrolysis is carried out at elevated pressure, see Figure 2, the conversion is even lower. When the pressure is decreased, the product composition shifts from alkanes to alkenes to alkynes, $C_{2}^{\prime}$ 's being the main products, when the pressure is decreased. $C_{3}$ hydrocarbons are only present in small amounts. 


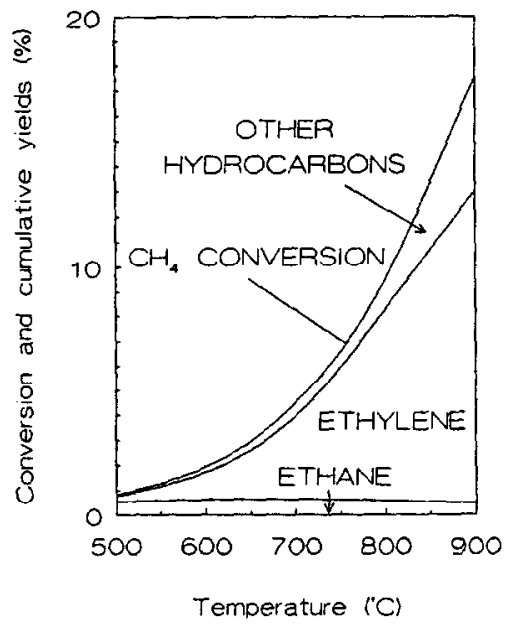

Figure 1 Calculated equilibrium gas composition of methane pyrolysis, excluding the formation of elementary carbon. $P=1$ bar.

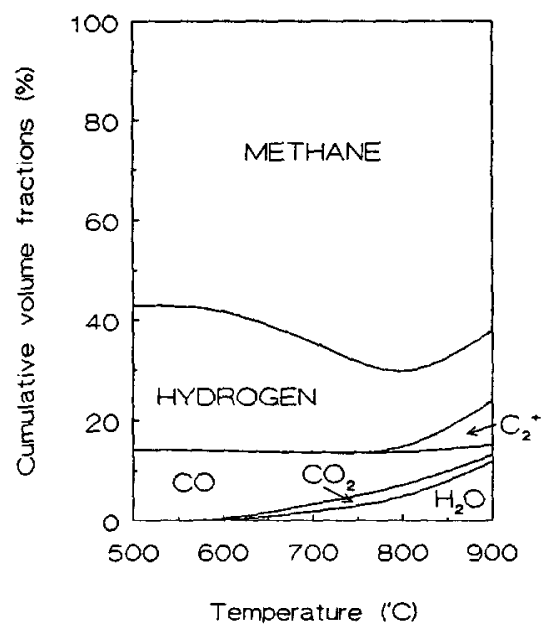

Figure 3 Calculated equilibrium composition for combined oxidation and pyrolysis of methane. $\mathrm{CH}_{4} / \mathrm{O}_{2}=10, \mathrm{P}=1$ bar.

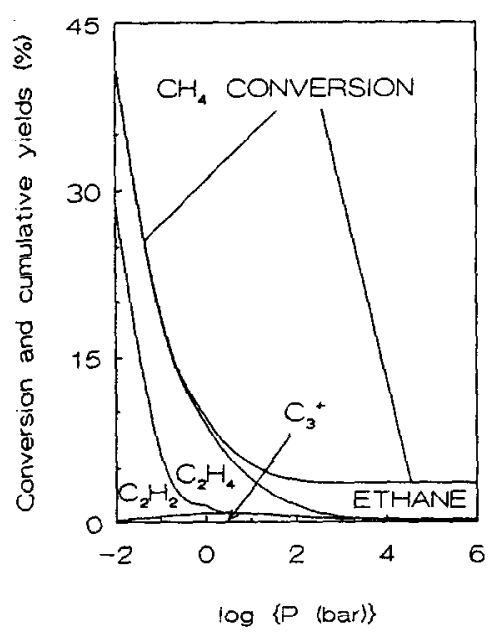

Figure 2 Calculated influence of the pressure on the equilibrium composition for methane pyrolysis. $\mathrm{T}=800^{\circ} \mathrm{C}$.

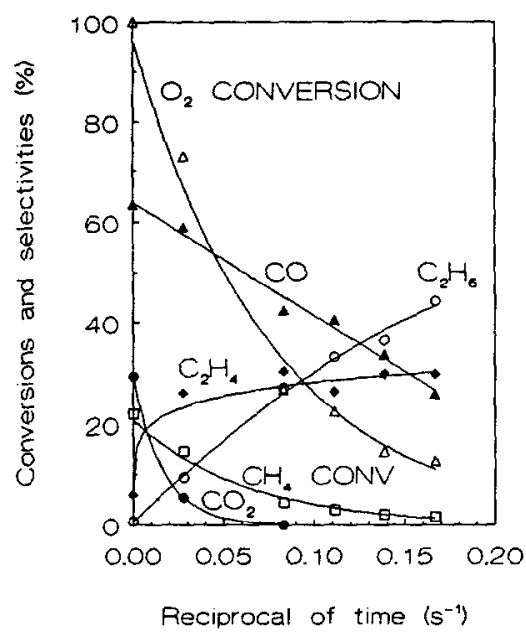

Figure 4 Influence of the residence time. Gas phase methane oxidation. $\mathrm{P}=1$ bar, $\mathrm{T}=800^{\circ} \mathrm{C}, \mathrm{CH}_{4} / \mathrm{O}_{2}=5$, $\mathrm{CH}_{4} / \mathrm{He}=0.8$.

When oxygen is added, the methane conversion is much higher, due to the high Gibbs free reaction energy of oxygen with hydrocarbons. In the equilibrium state the oxygen conversion is always complete when hydrocarbons are in excess. A cumulative volume fraction diagram of equilibrium compositions (initial $\mathrm{CH}_{4} / \mathrm{O}_{2}=10$ ) is plotted in Figure 3. At 
moderate temperatures the methane is converted into hydrogen and carbon monoxide. At higher temperatures, however, the products include $\mathrm{CO}_{2}$, water and hydrocarbons, with poor yields of the latter.

Gas phase experiments at varying residence time, Figure 4, show that kinetics govern the product distribution. The experiments were carried out in a quartz reactor of a micro flow reactor set-up, described elsewhere (ref. 1). Short contact times give high selectivities with respect to the desired $\mathrm{C}_{2} \mathrm{H}_{4}$ and $\mathrm{C}_{2} \mathrm{H}_{6}$, but at poor conversions, whereas long residence times (left part of the figure) favours the formation of carbon oxides and high conversions. The oxidation of $\mathrm{CO}$ to $\mathrm{CO}_{2}$ in the gas phase (ref. 2) is rather slow, which explains the very long residence times required to get carbon dioxide. Note that the points on the vertical axis, representing the calculated chemical equilibrium state, are covered very nicely by extrapolations of the experimental values. In agreement with the assumption that the formation of elementary carbon was negligible and could be omitted in the calculations, no carbon deposits were detected as long as oxygen was present in the outlet stream of the reactor.

\section{Model}

As is shown in Figure 4, oxidative coupling of methane can occur in the homogeneous gas phase. A computer program has been developed to simulate and analyze those reactions. The program is based on a combination of a reaction and a reactor model. The latter is modelled as an one dimensional ideal plug flow reactor. The gas phase reactions take place according to a complex free radical mechanism which can be described by a set of elementary reactions, see Table 1 . Each of these reactions is characterized by an extended Arrhenius expression for the reaction rate constant: $k=A \cdot T^{b} \cdot \exp (-E a c t / R T)$

$\begin{array}{lllll}\text { In which } & \mathbf{A} & = & \text { frequency factor } & \\ & \mathbf{b} & = & \text { non-linearity coefficient } & {[-]} \\ \mathrm{T} & =\text { absolute temperature } & {[\mathrm{K}]} \\ & \text { Eact } & =\text { energy of activation } & {[\mathrm{kJ} / \mathrm{mol}]} \\ \mathrm{R} & =\text { gas constant } & {[\mathrm{kJ} /(\mathrm{mol} \mathrm{K})]} \\ & \mathrm{AT} & \text { for a first order reaction } & {\left[\mathrm{s}^{-1}\right]} \\ & & \text { for a second order reaction } & {\left[\mathrm{m}^{3} /(\mathrm{mols})\right]} \\ & \text { for a third order reaction } & {\left[\mathrm{m}^{6} /\left(\mathrm{mol}^{2} \mathrm{~s}\right)\right]}\end{array}$

An important feature of the model developed is its option for a kinetic sensitivity analysis. It determines the influence of each elementary reaction on the product distribution. The principle of the method is that the frequency factor of each reaction in turn is divided by a constant, for example 10, and that new concentrations resulting from the calculations are used to compute integral sensitivity factors. These are defined as $s_{i j}=\Delta \ln c_{i} / \Delta \ln k_{j}$, in which 


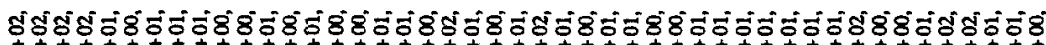

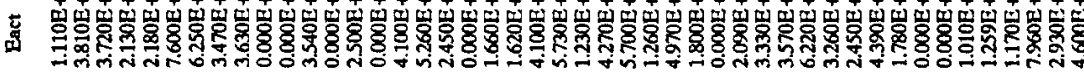

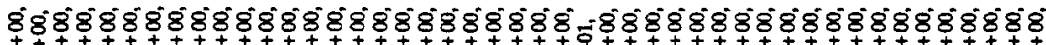

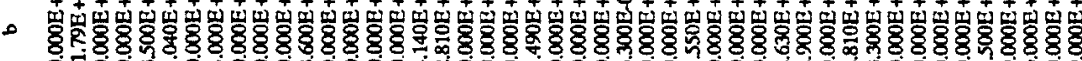
(4)

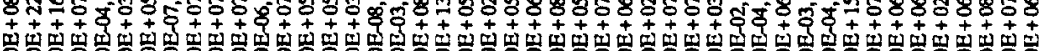

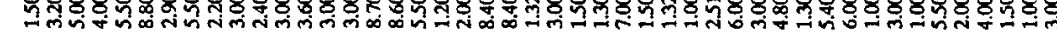

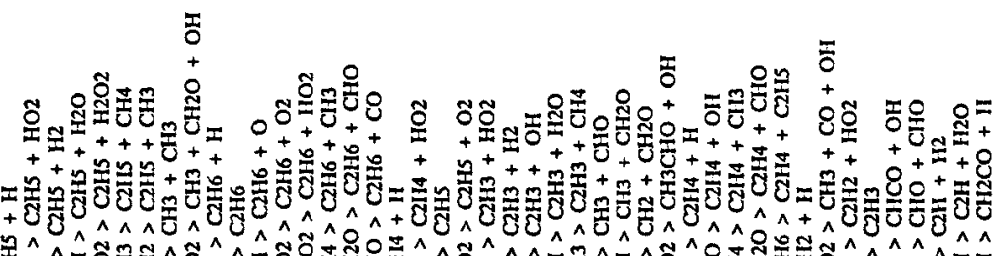

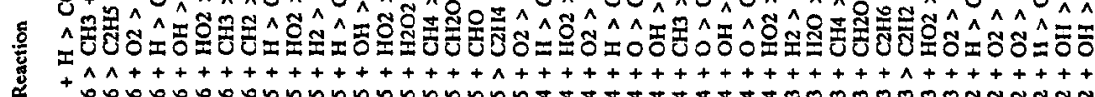
$+\hat{1} \hat{0}++++++++++++++++\hat{+}++++++++++++++++++1++++++++$

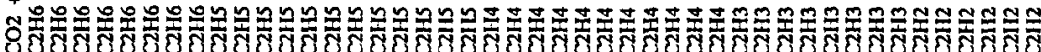

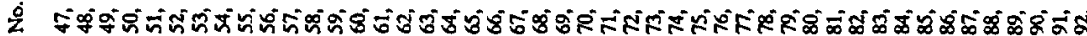

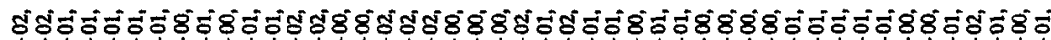

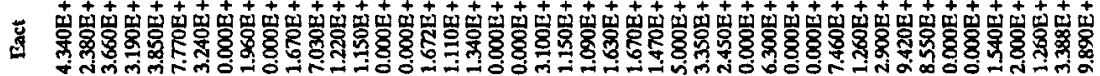

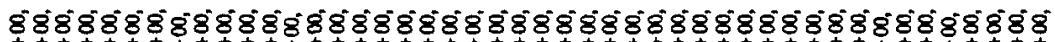

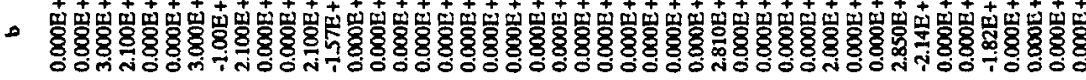
जิ

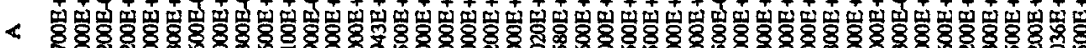

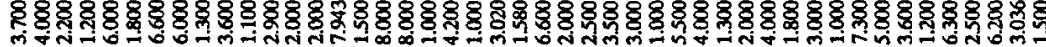

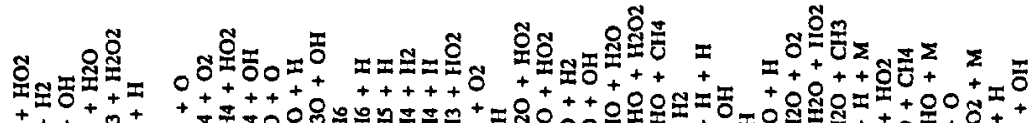

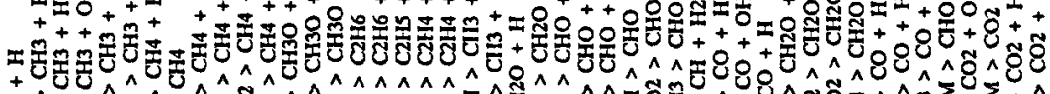

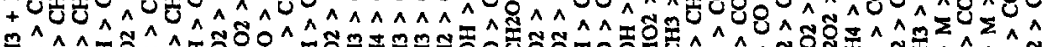

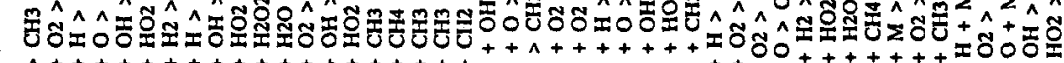

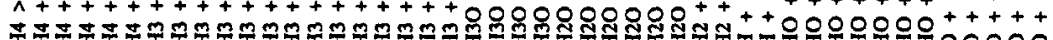

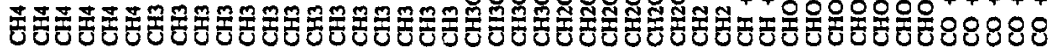

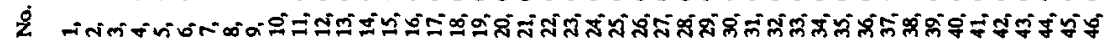


No. Reaction

93, $\mathrm{C}_{2} \mathrm{H}_{2}+\mathrm{CH}_{3}>\mathrm{C}_{2} \mathrm{H}+\mathrm{CH}_{4}$

94, $\mathrm{C} 2 \mathrm{H}+\mathrm{H}_{2}>\mathrm{C}_{2} \mathrm{H}_{2}+\mathrm{H}$

95, $\mathrm{C} 2 \mathrm{H}+\mathrm{H}_{2} \mathrm{O}>\mathrm{C}_{2} \mathrm{H} 2+\mathrm{OH}$

96, $\mathrm{C} 2 \mathrm{H}+\mathrm{CH}_{4}>\mathrm{C}_{2} \mathrm{H}_{2}+\mathrm{CH}_{3}$

97. $\mathrm{C} 2 \mathrm{H}+\mathrm{C}_{2} \mathrm{H} 6>\mathrm{C} 2 \mathrm{H} 2+\mathrm{C} 2 \mathrm{H} 5$

98, $\mathrm{C} 2 \mathrm{H} 2+\mathrm{O}>\mathrm{CH} 2+\mathrm{CO}$

99, $\mathrm{C} 2 \mathrm{H} 2+\mathrm{O}>\mathrm{CHCO}+\mathrm{H}$

$100, \mathrm{C} 2 \mathrm{H}+\mathrm{O} 2>\mathrm{CHO}+\mathrm{CO}$

$101, \mathrm{C} 2 \mathrm{H}+\mathrm{O}>\mathrm{CH}+\mathrm{CO}$

$102, \mathrm{CH} 3 \mathrm{CHO}+\mathrm{H}>\mathrm{CH} 3+\mathrm{CO}+\mathrm{H}_{2}$

$103, \mathrm{CH} 3 \mathrm{CHO}+\mathrm{O}>\mathrm{CH} 3+\mathrm{CO}+\mathrm{OH}$

$104, \mathrm{CH} 3 \mathrm{CHO}+\mathrm{OH}>\mathrm{CH} 3+\mathrm{CO}+\mathrm{H}_{2} \mathrm{O}$

$105, \mathrm{CH} 3 \mathrm{CHO}>\mathrm{CH} 3+\mathrm{CHO}$ $105, \mathrm{CH} 3 \mathrm{CHO}>\mathrm{CH} 3+\mathrm{CHO}+2.000 \mathrm{E}+15,0.000 \mathrm{E}+00$,

$107 . \mathrm{CH} 3 \mathrm{CHO}+\mathrm{O}_{2}>\mathrm{CH}_{3}+\mathrm{CO}+\mathrm{HO} 22.000 \mathrm{E}+07,5.000 \mathrm{E}-01$, $108, \mathrm{CH} 3 \mathrm{CHO}+\mathrm{CH} 3>\mathrm{CH} 3+\mathrm{CO}+\mathrm{CH} 4 \quad 8.000 \mathrm{E}+05,0.000 \mathrm{E}+\infty$,

$109, \mathrm{CH} 2 \mathrm{CO}+\mathrm{H}>\mathrm{CH} 3+\mathrm{CO}$

$110 \mathrm{CH} 2 \mathrm{CO}+\mathrm{O}>\mathrm{CHO}+\mathrm{CHO}$

$111, \mathrm{CH} 2 \mathrm{CO}+\mathrm{OH}>\mathrm{CHO}+\mathrm{CH} 2 \mathrm{O}$

$112, \mathrm{CH} 2 \mathrm{CO}+\mathrm{M}>\mathrm{CH} 2+\mathrm{CO}+\mathrm{M}$

$113, \mathrm{CHCO}+\mathrm{H}>\mathrm{CO}+\mathrm{CH} 2$

$114 \mathrm{CHCO}+\mathrm{O}>\mathrm{CO}+\mathrm{CHO}$

$115, \mathrm{CHCO}+\mathrm{OH}>\mathrm{CHO}+\mathrm{CO}+\mathrm{H}$

$116, \mathrm{CHCO}+\mathrm{O} 2>\mathrm{CO}+\mathrm{CO}+\mathrm{OH}$

$117, \mathrm{C} 2 \mathrm{HS}+\mathrm{CH}_{3}>\mathrm{C} 3 \mathrm{H} 8$

$118, \mathrm{C}_{2} \mathrm{H} 4+\mathrm{CH} 3>\mathrm{C}_{3} \mathrm{H} 7$

$119, \mathrm{C} 2 \mathrm{H} 3+\mathrm{CH}_{3}>\mathrm{C} 3 \mathrm{H} 6$

$120, \mathrm{C}_{2} \mathrm{H} 2+\mathrm{CH} 3>\mathrm{C} 3 \mathrm{H} 5$

$121, \mathrm{C} 3 \mathrm{H} 8>\mathrm{C}_{2} \mathrm{HS}+\mathrm{CH}_{3}$

$122, \mathrm{C} 3 \mathrm{H} 8+\mathrm{H}>\mathrm{C} 3 \mathrm{H} 7+\mathrm{H}_{2}$

$123, \mathrm{C} 3 \mathrm{H} 8+\mathrm{O}>\mathrm{C} 3 \mathrm{H}_{7}+\mathrm{OH}$

$124, \mathrm{C} 3 \mathrm{H} 8+\mathrm{OH}>\mathrm{C} 3 \mathrm{H} 7+\mathrm{H} 2 \mathrm{O}$

$125, \mathrm{C} 3 \mathrm{HB}+\mathrm{CH} 3>\mathrm{C} 3 \mathrm{H} 7+\mathrm{CH}_{4}$

$126, \mathrm{C}_{3} \mathrm{H} 7>\mathrm{C} 2 \mathrm{H} 4+\mathrm{CH} 3$

$127, \mathrm{C} 3 \mathrm{H} 7+\mathrm{H} 2>\mathrm{C} 3 \mathrm{H} 8+\mathrm{H}$

$128, \mathrm{C} 3 \mathrm{H} 7+\mathrm{H}>\mathrm{C} 3 \mathrm{H} 8$

$129, \mathrm{C} 3 \mathrm{H} 7+\mathrm{HO} 2>\mathrm{C} 3 \mathrm{H} 8+\mathrm{O}_{2}$

$130, \mathrm{C} 3 \mathrm{H} 7+\mathrm{CH}_{4}>\mathrm{C}_{3} \mathrm{H} 8+\mathrm{CH}_{3}$

$131, \mathrm{C} 3 \mathrm{H} 7+\mathrm{C} 2 \mathrm{H} 6>\mathrm{C} 3 \mathrm{H} 8+\mathrm{C} 2 \mathrm{H} 5$

$132, \mathrm{C} 3 \mathrm{H} 7+\mathrm{C} 2 \mathrm{H} 4>\mathrm{C} 3 \mathrm{H} 8+\mathrm{C}_{2} \mathrm{H}_{3}$

$133, \mathrm{C} 3 \mathrm{H} 7>\mathrm{C} 3 \mathrm{H} 6+\mathrm{H}$

$134, \mathrm{C} 3 \mathrm{H} 7+\mathrm{O} 2>\mathrm{C} 3 \mathrm{H} 6+\mathrm{HO} 2$

$135, \mathrm{C} 3 \mathrm{H} 6+\mathrm{H}>\mathrm{C} 3 \mathrm{H} 7$

$136, \mathrm{C} 3 \mathrm{H} 6>\mathrm{C} 2 \mathrm{H} 3+\mathrm{CH} 3$

$137, \mathrm{C} 3 \mathrm{H} 6+\mathrm{CH} 3>\mathrm{C} 3 \mathrm{H} 5+\mathrm{CH}_{4}$

$138, \mathrm{C}_{\mathrm{H}}+\mathrm{O}>\mathrm{C} \mathrm{CH}+\mathrm{CHO}$

$139, \mathrm{C} 3 \mathrm{H} 6+\mathrm{OH}>\mathrm{CH} 3 \mathrm{CHO}+\mathrm{CH}_{3}$

$140, \mathrm{C} 3 \mathrm{HS}+\mathrm{H} 2>\mathrm{C} 3 \mathrm{H} 6+\mathrm{H}$

$141, \mathrm{O} 2+\mathrm{H}>\mathrm{O}+\mathrm{OH}$

$142, \mathrm{O} 2+\mathrm{H}+\mathrm{M}>\mathrm{HO} 2+\mathrm{M}$

$143, \mathrm{O}+\mathrm{OH}>\mathrm{O} 2+\mathrm{H}$

$144, \mathrm{O}+\mathrm{HO} 2>\mathrm{O} 2+\mathrm{OH}$

$145, \mathrm{O}+\mathrm{H} 2>\mathrm{OH}+\mathrm{H}$

$146, \mathrm{O}+\mathrm{H} 2 \mathrm{O}>\mathrm{OH}+\mathrm{OH}$

$147, \mathrm{O}+\mathrm{H} 2 \mathrm{O} 2>\mathrm{OH}+\mathrm{HO} 2$

$148, \mathrm{OH}+\mathrm{H}>\mathrm{O}+\mathrm{H} 2$

$149, \mathrm{OH}+\mathrm{OH}>\mathrm{O}+\mathrm{H} 2 \mathrm{O}$

$150, \mathrm{OH}+\mathrm{H} 2>\mathrm{H} 2 \mathrm{O}+\mathrm{H}$

$151, \mathrm{OH}+\mathrm{H}+\mathrm{M}>\mathrm{H} 2 \mathrm{O}+\mathrm{M}$

$152, \mathrm{OH}+\mathrm{HO}_{2}>\mathrm{H} 2 \mathrm{O}+\mathrm{O}_{2}$

$153, \mathrm{OH}+\mathrm{H} 2 \mathrm{O} 2>\mathrm{H} 2 \mathrm{O}+\mathrm{HO} 2$

$154, \mathrm{HO} 2+\mathrm{M}>\mathrm{O} 2+\mathrm{H}+\mathrm{M}$

$155, \mathrm{HO} 2+\mathrm{H}>\mathrm{O} 2+\mathrm{H}_{2}$

$156, \mathrm{HO} 2+\mathrm{HO}_{2}>\mathrm{O}_{2}+\mathrm{OH}+\mathrm{OH}$

$157, \mathrm{HO} 2+\mathrm{H} 2>\mathrm{H}_{2} \mathrm{O} 2+\mathrm{H}$

$158, \mathrm{H} 2 \mathrm{O} 2+\mathrm{H}>\mathrm{HO} 2+\mathrm{H} 2$

$159, \mathrm{OH}+\mathrm{OH}+\mathrm{M}>\mathrm{H} 2 \mathrm{O} 2+\mathrm{M}$

$160, \mathrm{HO} 2+\mathrm{H}>\mathrm{OH}+\mathrm{OH}$

$161, \mathrm{H} 2 \mathrm{O} 2+\mathrm{M}>\mathrm{OH}+\mathrm{OH}+\mathrm{M}$

$162, \mathrm{H} 2 \mathrm{O} 2+\mathrm{H}>\mathrm{OH}+\mathrm{H} 2 \mathrm{O}$

$163, \mathrm{H} 2 \mathrm{O}+\mathrm{H}>\mathrm{OH}+\mathrm{H} 2$

$164, \mathrm{H}+\mathrm{H}+\mathrm{M}>\mathrm{H} 2+\mathrm{M}$

$M=$ inert
A

b

Eact

$1.800 \mathrm{E}+05, \quad 0.000 \mathrm{E}+\infty 0$ $3.500 \mathrm{E}+06, \quad 0.000 \mathrm{E}+00$ $4.070 \mathrm{E}+06, \quad 0.000 \mathrm{E}+00$, $1.000 \mathrm{E}+06, \quad 0.000 \mathrm{E}+00$ $3.600 E+06,0.000 E+\infty 0$ $1.750 \mathrm{E}+07, \quad 0.000 \mathrm{E}+00$ $9.000 E+06, \quad 0.000 \mathrm{E}+00$ $5.000 \mathrm{E}+07, \quad 0.000 \mathrm{E}+\infty 0$ $1.000 \mathrm{E}+07,0.000 \mathrm{E}+00$ $4.000 \mathrm{E}+07, \quad 0.000 \mathrm{E}+00$ $5.000 \mathrm{E}+06, \quad 0.000 \mathrm{E}+\infty$ $1.000 \mathrm{E}+07, \quad 0.000 \mathrm{E}+00$ $2.000 \mathrm{E}+07,0.000 \mathrm{E}+\infty$ $1.000 E+07, \quad 0.000 E+00$ $1.000 \mathrm{E}+10, \quad 0.000 \mathrm{E}+\infty 0$, $3.000 \mathrm{E}+06, \quad 0.000 \mathrm{E}+00$, $1.200 \mathrm{E}+06, \quad 0.000 \mathrm{E}+00$, $1.000 \mathrm{E}+07, \quad 0.000 \mathrm{E}+00$ $1.460 \mathrm{E}+06, \quad 0.000 \mathrm{E}+00$, $1.000 \mathrm{E}+07, \quad 0.000 \mathrm{E}+00$ $1.000 \mathrm{E}+05, \quad 0.000 \mathrm{E}+00$ $1.000 \mathrm{E}+07, \quad 0.000 \mathrm{E}+00$ $6.000 \mathrm{E}+05, \quad 0.000 \mathrm{E}+\infty 0$ $7.900 \mathrm{E}+16, \quad 0.000 \mathrm{E}+\infty 0$ $1.300 \mathrm{E}+08, \quad 0.000 \mathrm{E}+00$ $3.000 \mathrm{E}+07, \quad 0.000 \mathrm{E}+00$ $3.700 \mathrm{E}+06, \quad 0.000 \mathrm{E}+00$, $1.550 \mathrm{E}+08, \quad 0.000 \mathrm{E}+00$ $1.300 \mathrm{E}+13, \quad 0.000 \mathrm{E}+00$ $4.000 \mathrm{E}+06, \quad 0.000 \mathrm{E}+\infty 0$ $2.000 \mathrm{E}+07, \quad 0.000 \mathrm{E}+00$ $1.000 \mathrm{E}+07, \quad 0.000 \mathrm{E}+00$ $7.900 \mathrm{E}+05, \quad 0.000 \mathrm{E}+00$ $1.000 \mathrm{E}+05, \quad 0.000 \mathrm{E}+00$ $1.000 \mathrm{E}+05, \quad 0.000 \mathrm{E}+00$, $2.000 \mathrm{E}+14, \quad 0.000 \mathrm{E}+\infty 0$ $1.000 \mathrm{E}+06, \quad 0.000 \mathrm{E}+00$ $4.000 \mathrm{E}+06, \quad 0.000 \mathrm{E}+00$, $7.000 \mathrm{E}+16, \quad 0.000 \mathrm{E}+00$ $2.000 \mathrm{E}+04, \quad 0.000 \mathrm{E}+00$, $1.510 \mathrm{E}+06, \quad 0.000 \mathrm{E}+00$ $1.000 \mathrm{E}+07, \quad 0.000 \mathrm{E}+\infty 0$ $7.940 \mathrm{E}+06, \quad 0.000 \mathrm{E}+00$, $1.700 \mathrm{E}+11,-9.00 \mathrm{E}-01$ $2.000 \mathrm{E}+06,-8.00 \mathrm{E}-01$, $1.800 \mathrm{E}+07, \quad 0.000 \mathrm{E}+00$ $2.000 \mathrm{E}+07, \quad 0.000 \mathrm{E}+00$ $1.500 \mathrm{E}+01, \quad 2.000 \mathrm{E}+00$ 4.600E +03, 1.300E + 00 $6.310 \mathrm{E}+06, \quad 0.000 \mathrm{E}+00$ $6.700 \mathrm{E}+00, \quad 2.000 \mathrm{E}+00$ $1.500 \mathrm{E}+03, \quad 1.140 \mathrm{E}+00$ $1.000 \mathrm{E}+02, \quad 1.600 \mathrm{E}+\infty 0$ $2.200 \mathrm{E}+10,-2.00 \mathrm{E}+00$, $2.000 \mathrm{E}+07, \quad 0.000 \mathrm{E}+00$ $1.750 \mathrm{E}+06,0.000 \mathrm{E}+00$ $2.100 \mathrm{E}+09, \quad 0.000 \mathrm{E}+\infty 0$ $2.500 \mathrm{E}+07, \quad 0.000 \mathrm{E}+00$ $2.000 \mathrm{E}+06, \quad 0.000 \mathrm{E}+00$ $3.000 \mathrm{E}+07,0.000 \mathrm{E}+\infty 0$ $1.700 \mathrm{E}+06, \quad 0.000 \mathrm{E}+\infty$, $3.200 \mathrm{E}+10,-2.00 \mathrm{E}+00$, $1.500 \mathrm{E}+08, \quad 0.000 \mathrm{E}+00$ $3.000 \mathrm{E}+11, \quad 0.000 \mathrm{E}+00$ $1.000 \mathrm{E}+07, \quad 0.000 \mathrm{E}+00$ 4.600E + 02, $1.600 \mathrm{E}+\infty 0$ $1.800 \mathrm{E}+06,-1.00 \mathrm{E}+00$
$7.230 \mathrm{E}+01$, $8.800 \mathrm{E}+00$, $5.800 \mathrm{E}+00$, $6.000 \mathrm{E}+01$, $0.000 \mathrm{E}+00$, $1.330 \mathrm{E}+01$, $1.900 \mathrm{E}+01$, $6.300 \mathrm{E}+00$, $0.000 \mathrm{E}+00$, $1.760 \mathrm{E}+01$, $7.500 \mathrm{E}+00$, $0.000 \mathrm{E}+00$, $3.310 \mathrm{E}+02$, $4.480 \mathrm{E}+01$, $1.765 \mathrm{E}+02$, $2.510 \mathrm{E}+01$, $1.260 \mathrm{E}+01$, $9.600 \mathrm{E}+00$, $0.000 \mathrm{E}+00$, $2.480 \mathrm{E}+02$, $0.000 \mathrm{E}+00$, $0.000 \mathrm{E}+00$, $0.000 \mathrm{E}+00$, $1.050 E+01$, $0.000 \mathrm{E}+00$, $2.900 \mathrm{E}+01$, $0.000 \mathrm{E}+00$, $3.220 \mathrm{E}+01$, $3.560 \mathrm{E}+02$, $4.060 \mathrm{E}+01$, $2.410 \mathrm{E}+01$, $6.900 \mathrm{E}+00$, $8.900 \mathrm{E}+01$, $1.359 \mathrm{E}+02$, $6.980 \mathrm{E}+01$, $0.000 \mathrm{E}+00$, $0.000 \mathrm{E}+00$, $6.480 \mathrm{E}+01$, $4.180 \mathrm{E}+01$, $6.280 \mathrm{E}+01$, $1.630 \mathrm{E}+02$, $2.090 \mathrm{E}+01$, $1.100 \mathrm{E}+01$, $3.600 \mathrm{E}+02$, $1.880 \mathrm{E}+01$, $0.000 \mathrm{E}+\infty 0$, $0.000 \mathrm{E}+00$, $3.100 \mathrm{E}+01$, $7.280 \mathrm{E}+01$, $0.000 \mathrm{E}+00$ $0.000 \mathrm{E}+\infty$, $0.000 \mathrm{E}+00$, $3.160 \mathrm{E}+01$, $7.150 \mathrm{E}+01$, $2.075 \mathrm{E}+01$, $2.330 \mathrm{E}+01$, $0.000 \mathrm{E}+00$, $1.380 \mathrm{E}+01$, $0.000 \mathrm{E}+00$, $0.000 \mathrm{E}+\infty$, $1.330 \mathrm{E}+\infty 0$ $1.910 \mathrm{E}+02$, $2.900 \mathrm{E}+\infty$, $0.000 \mathrm{E}+00$, $1.090 \mathrm{E}+02$, $1.570 \mathrm{E}+01$ $0.000 \mathrm{E}+00$, $4.200 \mathrm{E}+00$, $1.900 \mathrm{E}+02$, $1.660 \mathrm{E}+01$, $7.770 \mathrm{E}+01$, $0.000 \mathrm{E}+\infty 0$ $c_{i}=$ concentration of component $i$ and $k_{j}=$ rate constant of reaction $j$. A set of over 400 reactions, not shown here, could be reduced to the 164 reactions of Table 1 by omitting all the reactions which had no significant contribution to the product distribution. In this way, for example the formation of methanol and reactions in which a methyldioxy radical is involved were skipped. This simplifies the reaction network but at the same time limits the applicability to conditions relevant for the methane oxidative coupling. The product distribution turns out to be highly sensitive towards reactions no. $6,13,15,16,46$, 54 , and 70 . A reasonable fit between the model and experimental data (ref. 15) was obtained (see Figure 5), using the kinetic constants from the literature. Only minor changes (within reported accuracy limits) were applied for some of the most sensitive constants. Note that the ethylene selectivity predicted by the model is too low. This requires further attention. The negative ethylene selectivities at low methane conversion are caused 


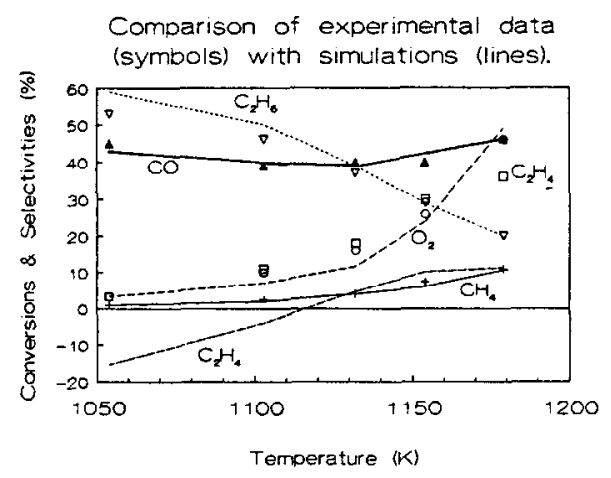

Figure 5 Comparison of experiments (symbols) with simulations (lines).

Conditions: $\mathrm{CH}_{4} / \mathrm{O}_{2}=10, \mathrm{CH}_{4} / \mathrm{He}=0.8, \mathrm{P}=1$ bar.

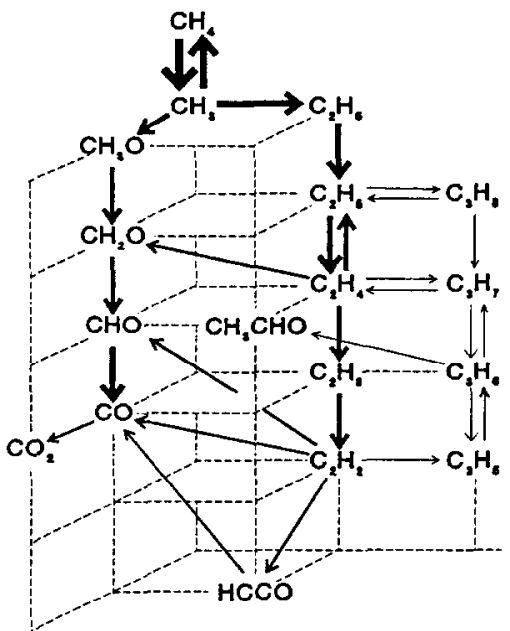

Figure 6 The simplified gas phase reaction scheme with the main reaction paths. The width of an arrow indicates the importance of that reaction path.

Conditions: $\mathrm{T}=800^{\circ} \mathrm{C}, \mathrm{P}=1 \mathrm{bar}, \mathrm{CH}_{4} / \mathrm{O}_{2}=5$.

by (calculated) conversion of traces of ethylene present in the feed.

Another powerful tool of the model is the integration of individual reaction rates over the reactor, thus demonstrating the most important reaction pathways. It appears that $\mathrm{H}, \mathrm{OH}$, $\mathrm{HO}_{2}$, and $\mathrm{CH}_{3}$ are the most reactive species. After an induction period in which the radical concentrations are built up, they stay fairly constant at typical concentrations of $10^{-7}-10^{-4}$ $\mathrm{mol} / \mathrm{m}^{3}$. Figure 6 shows the main reactants and reaction pathways of carbon containing compounds. The thickness of the arrows corresponds to the contribution of the respective reaction. It may be obvious that the $\mathrm{C}_{2}$-oxygenates, $\mathrm{CH}_{2}, \mathrm{CH}, \mathrm{CH}_{3} \mathrm{OH}$ and other omitted species are unimportant. The most important initiation reaction no. 2 , in which oxygen reacts with methane, appears to contribute very little to the methane conversion, and the same applies to other initiation reactions. The formation of $\mathrm{CH}_{3}$ radicals from methane appears in the scheme as a rapid system of equilibrium reactions in which various radicals are involved. In agreement with experimental results, the quantities of $\mathrm{C}_{3}$-products are very limited, not because they are oxidised very fast, but because of their low rate of formation, see Figure 6. The $\mathrm{C}_{2}$-species prefer to undergo hydrogen abstraction rather than coupling with methyl radicals. Ethane and ethyl radicals are hardly oxidised and the formation of $\mathrm{CH}_{3}$ from $\mathrm{C}_{2} \mathrm{H}_{6}$ is negligably small, which implies that extremely high initial ethylene selectivity of ethane oxidation should be achievable. This is confirmed, although not shown here, experimentally. 
The trends of the effects of temperature, dilution and $\mathrm{CH}_{4} / \mathrm{O}_{2}$ on the selectivity of the reaction are very similar for both homogeneous gas phase reactions and the $\mathrm{Li} / \mathrm{MgO}$ catalyzed reactions. We therefore assume that the reaction scheme is valuable for the catalytic reactions as well. A major TABLE 2

difference is the formation of $\mathrm{CH}_{3}$

Experiments and simulations of both catalytic and

radicals, the key component for a good

homogeneous gas phase reactions. Reactor made of quartz.

Conditions: $\mathrm{T}=800^{\circ} \mathrm{C}, \mathrm{P}=1 \mathrm{bar}, \mathrm{CH}_{4} / \mathrm{O}_{2}=10$.

$\mathrm{C}_{2}{ }^{+}$selectivity, see Figure 6. A catalyst favours the (irreversible) formation of this radical (ref 3.), involving oxygen and resulting in much higher levels of $\mathrm{CH}_{3}$ radicals. Thus higher rates and higher coupling efficiencies may be achieved using a catalyst. In Table 2 simulations
Gas Phase $\mathrm{Li} / \mathrm{MgO}$ catalyst Exp. Sim. Exp. Sim.

$\begin{array}{lllll}\text { Time [s] } & 4.6 & 7.9 & 6.0 & 0.5 \\ \mathrm{O}_{2} \text {-conv [\%] } & 28.2 & 28.2 & 92.8 & 92.8 \\ \mathrm{CH}_{4} \text {-conv [\%] } & 4.1 & 4.2 & 13.4 & 20.2 \\ \mathrm{C}_{2} \text { sel [\%] } & 51.1 & 49.7 & 76.2 & 76.9 \\ \mathrm{CO}_{x^{s e l}}[\%] & 48.8 & 50.0 & 23.8 & 22.8 \\ \mathrm{CH}_{3}\left[\mathrm{~mol} / \mathrm{m}^{3}\right] & -\cdots & 5.9 \mathrm{E}-4 & --- & 2.3 \mathrm{E}-3\end{array}$
and experiments are shown, for both catalyzed and homogeneous gas phase reactions. The catalyst is simulated by a set of reactions that equals the overall reaction: $4 \mathrm{CH}_{4}+\mathrm{O}_{2} \ldots$ $4 \mathrm{CH}_{3}+2 \mathrm{H}_{2} \mathrm{O}$. As is shown, indeed the higher $\mathrm{CH}_{3}$ radical formation rate results in a higher reaction rate and a higher ethane selectivity. The decrease in reaction time is however too large compared to the experimental value. Therefore, it is assumed that the catalyst also acts as a radical sink. Radicals collide with the surface and react to less active molecules. This lowers the radical concentrations and slows down the conversions of methane and oxygen.

\section{CONCLUSIONS}

In the calculated chemical equilibrium state, ethylene is the main product of methane pyrolysis when the formation of elementary carbon is excluded. The ethylene yield however is limited, due to a limited methane conversion that even gets worse when the pressure is increased. Addition of oxygen leads to a much higher methane conversion, however at low $\mathrm{C}_{2}{ }^{+}$selectivities. Extrapolation of the results of gas phase experiments to the condition of infinite residence time gives an excellent fit with values calculated for the equilibrium state.

The model developed simulates the experiments correctly. The main carbon reaction path goes via $\mathrm{CH}_{3}, \mathrm{C}_{2} \mathrm{H}_{6}, \mathrm{C}_{2} \mathrm{H}_{5}$, towards $\mathrm{C}_{2} \mathrm{H}_{4}$, that is oxidised rapidly into $\mathrm{CO}$. The most active reaction species in the homogeneous gas phase are the radicals $\mathrm{H}, \mathrm{OH}, \mathrm{CH}_{3}$ and $\mathrm{OH}_{2} . \mathrm{C}_{3}$ hydrocarbons are hardly produced due to their low rate of formation. Simulations have made clear that the $\mathrm{Li} / \mathrm{MgO}$ catalyst cannot be described as a methyl radical producer only: probably it also acts as an (aselective) radical consumer. 


\section{ACKNOWLEDGEMENT}

The financial support for this research, which was provided by the European Communities under contract no. EN3C-0038-NL, and by the Netherlands Organization for the Advancement of Pure Research, is gratefully acknowledged.

\section{REFERENCES}

1 J.M.N. van Kasteren, J.W.M.H. Geerts, K. van der Wiele, Ethylene synthesis by catalytic oxidation of methane, in: M.J. Phillips (Ed.), Proc. $9^{\text {th }}$ ICC, Calgary, Canada, 1988, pp. 930-936.

2 J.W.M.H. Geerts, J.M.N. van Kasteren, K. van der Wiele, The investigation of individual reaction steps in the oxidative coupling of methane over lithium doped magnesium oxide, Cat. Today, 4 (1989) 453-461.

3 D.J. Driscoll, J.H. Lunsford, Gas-Phase radical formation during the reactions of methane, ethane, ethylene and propylene over selected oxide catalysts, J/ Phys. Chem., 89 (1985), 4415-4418.

4 W. Tsang, R.F. Hampsen, Chemical Kinetic Data Base for Combustion Chemistry, Part 1 Methane and related compounds, 1986, pp 1087-1279.

5 W.C. Gardiner (Ed.), Combustion Chemistry, Springler-Verlag, New York Inc., 1984, pp. 197-360.

6 F. Westley, J.T. Herron, Compilation of Kinetic Data for Combustion Chemistry, Part 1 Non aromatic C,H,O,N and S containing compounds, 1987.

7 J. Warnatz, Hydrocarbon Oxidation at High Temperatures, Ber. Bunsenges. Phys. Chem. 87 (1983) 1008-1022.

8 I.A. Vardanyan, A.B. Nalbandyan, On the mechanism of thermal Oxidation of Methane, Int. J. Chem. Kin., 17 (1985) 901-924.

9 P. Dagaut, M. Cathonnet, J.C. Boettner, Experimental Study and Kinetic Modeling of Propene Oxidation in a jet Stirred Flow Reactor, J. Phys. Chem., (1988) 661-671.

$10 \mathrm{G}$. Rotzoll, Mass Spectrometric Investigation and Computer Modeling of $\mathrm{CH}_{4}-\mathrm{O}_{2}-\mathrm{O}_{3}$ Reaction from 480 to $830 \mathrm{~K}$, J. Phys. Chem., 90 (1986) 677-683.

11 H.D. Gesser, N.R. Hunter, C.B. Prakash, The Direct Conversion of Methane to Methanol by controlled Oxidation, Chemical Reviews, 85 (4) (1985) 235-244.

$12 \mathrm{~J}$. Nicholas, Chemical Kinetics, A modern Survey of Gas Reactions, Harper \& Row Ltd, London, 1976.

13 D.L. Baulch, D.D. Drysdale, D.G. Horne and A.C. Lloyd, Evaluated Kinetic Data for High Temperature Reactions, Butterworths, London, 1972.

14 L.F. Albright, B.L. Crynes, W.H. Corcoran, (Ed.), Pyrolysis, theory and industrial practice, Academic Press, 1983.

15J. Elbers, AKZO, personal communication. 